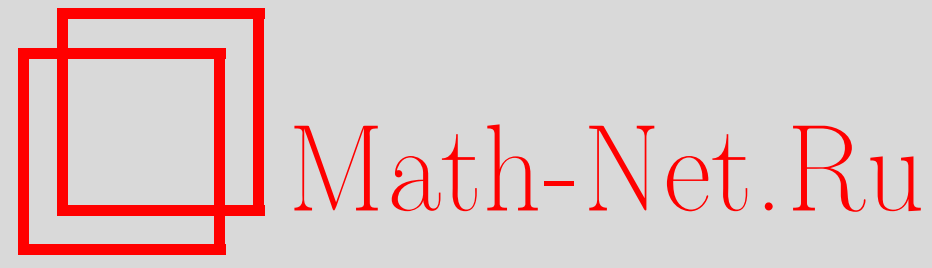

Общероссийский математический портал

А. А. Зайцев, K теории натуральных гамильтоновых систем, интегрируемых в эллиптических координатах, Матем. заметки, 1996, том 60, выпуск $6,924-929$

DOI: https://doi.org/10.4213/mzm1911

Использование Общероссийского математического портала MathNet.Ru подразумевает, что вы прочитали и согласны с пользовательским соглашением 
http://www . mathnet.ru/rus/agreement

Параметры загрузки:

IP : 18.207 .199 .55

26 апреля 2023 г., 12:54:31

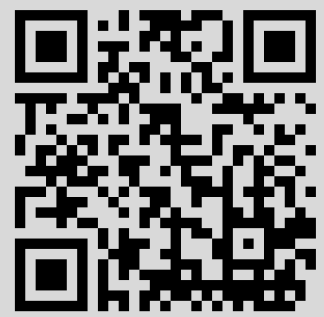




\section{К ТЕОРИИ НАТУРАЛЬНЫХ ГАМИЛЬТОНОВЫХ СИСТЕМ, ИНТЕГРИРУЕМЫХ В ЭЛЛИПТИЧЕСКИХ КООРДИНАТАХ}

\section{А. А. Зайцев}

1. Недавно Маршалл и Войцеховский указали [1] новое семейство натуральных гамилтоновых систем, которые интегрируются в эллиптических координатах разделением переменных. Генетически эти системы связаны с рациональными первыми интегралами геодезического потока на эллипсоиде $\left(\right.$ в $\left.\mathbb{R}^{n}\right) \sum_{i=1}^{n} x_{i}^{2} / a_{i}=1$, $0<a_{1}<a_{2}<\cdots<a_{n}$ (задача Якоби; см. [2]),

$$
f_{k}(x, p)=p_{k}^{2}+\sum_{i \neq k} \frac{\left(x_{k} p_{i}-x_{i} p_{k}\right)^{2}}{a_{k}-a_{i}}, \quad p_{k}=\dot{x}_{k},
$$

$x=\left(x_{1}, \ldots, x_{n}\right), p=\left(p_{1}, \ldots, p_{n}\right)$ (в скобках указаны декартовы координаты точек $x, p \in \mathbb{R}^{2 n} ;$ всюду в статье индексы $\left.i, k, m=\overline{1, n}\right)$. Они коммутируют в $\mathbb{R}^{n}$ и $\sum_{i} f_{i}=2 T=|p|^{2}$. Естественно дополнить их слагаемыми $2 u_{k}(x)$ так, чтобы функции

$$
F_{k}(x, p)=f_{k}(x, p)+2 u_{k}(x)
$$

снова попарно коммутировали, тогда они будут первыми интегралами гамильтонова потока $g_{h}^{t}$, где

$$
h=T+u(x), \quad u(x) \equiv \sum_{i} u_{i}(x)
$$

это приводит к новым интегрируемым системам с натуральными гамилтонианами.

Теорема 1 (Маршалл-Войцеховский [1]). Пусть функции $F_{k}(x, p)$ определены равенствами (2), тогда следующие условия эквивалентны:

а) они есть первые интеграль гамильтонова потока с гамильтонианом (3);

б) они образуют полный инволютивный набор первых интегралов; 
в) потенииал $и(x)$ удовлетворяет системе уравнений

$$
\begin{aligned}
& \left(a_{k}-a_{m}\right)^{-1}\left(x_{k}^{2} \frac{\partial^{2} u}{\partial x_{m} \partial x_{i}}-x_{k} x_{m} \frac{\partial^{2} u}{\partial x_{k} \partial x_{i}}\right) \\
& \quad=\left(a_{k}-a_{i}\right)^{-1}\left(x_{k}^{2} \frac{\partial^{2} u}{\partial x_{m} \partial x_{i}}-x_{k} x_{i} \frac{\partial^{2} u}{\partial x_{k} \partial x_{m}}\right), \quad i \neq k, \quad k \neq m \\
& \frac{\partial^{2} u}{\partial x_{k} \partial x_{m}}+3\left(a_{k}-a_{m}\right)^{-1}\left(x_{m} \frac{\partial u}{\partial x_{k}}-x_{k} \frac{\partial u}{\partial x_{m}}\right) \\
& -\left(a_{k}-a_{m}\right)^{-1}\left(x_{k}^{2} \frac{\partial^{2} u}{\partial x_{k} \partial x_{m}}-x_{k} x_{m} \frac{\partial^{2} u}{\partial x_{k}^{2}}\right) \\
& \quad+\sum_{i \neq k}\left(a_{k}-a_{i}\right)^{-1}\left(x_{i}^{2} \frac{\partial^{2} u}{\partial x_{k} \partial x_{m}}-x_{k} x_{i} \frac{\partial^{2} u}{\partial x_{m} \partial x_{i}}\right)=0, \quad k \neq m
\end{aligned}
$$

г) полный интеграл стационарного уравнения Гамильтона-Якоби получается разделением переменных в эллиптических координатах.

Анализ, данный в [1], полезно дополнить. Например, особый интерес представляют гамильтонианы (3) с полиномиальными потенциалами $u(x)$, так как они возникают в задачах о взаимодействии ангармонических осцилляторов и др. В частности, системы Маршалла-Войцеховского содержат случай $u(x)=\sum_{i} a_{i} x_{i}^{2}-|x|^{4}$, изученный Гарнье [3]. В [4] доказано, что решения системы Гарнье выражаются через собственные функции одномерного оператора Шрёдингера с конечнозначными потенциалами. В данной работе дается описание всех полиномиальных потенциалов в системах Маршалла-Войцеховского, приводится их производящая функция и производящая функция (по Мозеру) первых интегралов, излагается еще ряд фактов. Другой подход к проблеме и часть результатов изложены в заметке автора [5].

2. Так как функции $f_{k}$ коммутируют с кинетической энергией $T$, то все $F_{k}$ будут первыми интегралами гамильтонова потока $g_{h}^{t}$ тогда и только тогда, когда $2\left\{u_{k}(x), T\right\}=\left\{u(x), f_{k}(x, p)\right\}(\{\cdot, \cdot\}$ означает стандартную скобку Пуассона). Эти соотношения линейные по импульсам, поэтому расщепляются, давая систему уравнений

где

$$
\frac{\partial u_{k}}{\partial x_{k}}=l_{k} u, \quad \frac{\partial u_{k}}{\partial x_{m}}=x_{k} \sigma_{k m} u, \quad m \neq k,
$$

$$
\sigma_{k i} f \equiv\left(a_{k}-a_{i}\right)^{-1}\left(x_{k} \frac{\partial f}{\partial x_{i}}-x_{i} \frac{\partial f}{\partial x_{k}}\right), \quad i \neq k, \quad l_{k} f \equiv \frac{\partial f}{\partial x_{k}}-\sum_{i \neq k} x_{i} \sigma_{i k} f .
$$

Исключая из (5) $u_{k}$ перекрестными дифференцированиями, получаем для основного потенциала $u(x)$ систему (4). Заметим, что $\sigma_{k m}=\sigma_{m k} ;$ с помощью этого равенства доказьвается

ТЕОРема 2. Для решений системы (5) справедливы представления

$$
u_{k}=x_{k} \frac{\partial \varphi}{\partial x_{k}}, \quad u=\sum_{i} x_{i} \frac{\partial \varphi}{\partial x_{i}}
$$

әде функиия $\varphi$ удовлетворяет уравнениям

$$
\frac{\partial^{2} \varphi}{\partial x_{k} \partial x_{m}}=\sigma_{k m} \sum_{i} x_{i} \frac{\partial \varphi}{\partial x_{i}}, \quad k \neq m
$$


Простейшее решение системы (5) есть

$$
u_{k}=u_{k}^{(1)} \equiv x_{k}^{2}, \quad u=u^{(1)} \equiv|x|^{2} .
$$

Размножить решения позволяет

ТЕОРема 3. Если функиии $u_{k}$, и удовлетворяют системе (5), то преобразование

$$
\widetilde{u}_{k}=a_{k} u_{k}-x_{k}^{2} u, \quad \widetilde{u}=\sum_{i} \widetilde{u}_{i}
$$

дает новые решения этой системы.

Это утверждение остается, разумеется, справедливым, если новые потенциалы $\widetilde{u}_{k}$ дополнить произвольными константами. Итерируя решение (6) преобразованиями (7) в сочетании с операцией сложения с константами, получаем новые многочлены. Первая итерация дает потенциалы Гранье $u_{k}^{(2)}=x_{k}^{2}\left(a_{k}-|x|^{2}\right)$, $u^{(2)}=\sum_{i} a_{i} x_{i}^{2}-|x|^{4}$.

Обозначим через $u_{k}^{(\nu)}, u^{(\nu)}, \nu \in \mathbb{N}$, потенциалы, получаемые из потенциалов (6) последовательными итерациями преобразования (7); для них справедливы рекуррентные соотношения

$$
u_{k}^{(\nu+1)}=a_{k} u_{k}^{(\nu)}-x_{k}^{2} u^{(\nu)}, \quad u^{(\nu)}=\sum_{i} u_{i}^{(\nu)}, \quad \nu \in \mathbb{N}
$$

Используя теорему 2, можно установить, что старшие однородные члены любьх полиномиальных решений системы (5) есть $c_{\nu} x_{k}^{2}|x|^{2(\nu-1)}$ (для $u_{k}$ ) и $c_{\nu}|x|^{2 \nu}$ (для $u$ ). Следствием этого факта является

Теорема 4. Потенциальи $u_{k}^{(\nu)}, u^{(\nu)}, \nu \in \mathbb{N}$, образуют базис векторного пространства всех полиномиальных решений системы (5).

Многочлены, о которых идет речь в теореме 4, назовем базисными. Определим их производящие функции равенствами

$$
w_{k}(\lambda, x)=\sum_{\nu \in \mathbb{N}} u_{k}^{(\nu)} \lambda^{-\nu}, \quad w(\lambda, x)=\sum_{i} w_{i}(\lambda, x)=\sum_{\nu \in \mathbb{N}} u^{(\nu)} \lambda^{-\nu} .
$$

Значения производящих функций получаются умножением (8) на $\lambda^{-\nu}$ и суммированием по $\nu$; это дает

$$
w_{k}(\lambda, x)=\frac{x_{k}^{2}}{\left(\lambda-a_{k}\right)\left(1-Q_{\lambda}(x)\right)}, \quad w(\lambda, x)=-\frac{Q_{\lambda}(x)}{1-Q_{\lambda}(x)},
$$

где $Q_{\lambda}(x)=\sum_{i} x_{i}^{2} /\left(a_{i}-\lambda\right)$.

Обратное преобразование к преобразованию (7) дается равенством

$$
u_{k}=a_{k}^{-1} \frac{\widetilde{u}_{k}+x_{k}^{2} \sum_{i} \widetilde{u}_{i} / a_{i}}{1-Q_{0}(x)}
$$

Система (5) инвариантна относительно этого преобразования. Комбинируя его с прямьм преобразованием (7) и операцией сложения с произвольными константами, получаем многочисленное семейство рациональных решений системы (5). Вероятно, этот способ дает все рациональные решения. 
3. Эллиптические координаты $\left\{\xi_{k}\right\}$ точки $x \in \mathbb{R}^{n}$ определяются как корни уравнения $Q_{\lambda}(x)=1$ (относительно $\lambda$ ). Вычисляя вычет функции $Q_{\lambda}(x)$ в полюсе $\lambda=a_{k}$, получаем формулу [2]

$$
x_{k}^{2}=\frac{D\left(\xi_{k}\right)}{\prod_{i \neq k}\left(a_{k}-a_{i}\right)}, \quad D\left(\xi_{k}\right) \equiv \prod_{i}\left(\xi_{k}-a_{i}\right),
$$

которая выражает декартовы координаты точки $x$ через эллиптические. Эллиптическая система координат ортогональная: в ней евклидова метрика имеет вид (вывод основан на формуле (11)) $d s^{2}=\sum_{k} g_{k} d \xi_{k}^{2}$, где $g_{k}=-\prod_{i \neq k}\left(\xi_{k}-\xi_{1}\right) / 4 D\left(\xi_{k}\right)$. Диагональность метрического тензора равносильна тождеству

$$
\sum_{i} \frac{x_{i}^{2}}{\left(a_{i}-\xi_{k}\right)\left(a_{i}-\xi_{m}\right)}=4 g_{k} \delta_{k m}
$$

(здесь и далее под $x_{k}^{2}$ подразумевается выражение $(11)$ этой величины через эллиптические координаты), из которого следует

ТЕОРема 5. Линейные преобразования

$$
s_{m}=\sum_{k} \frac{c_{k}}{a_{k}-\xi_{m}}, \quad c_{k}=x_{k}^{2} \sum_{i} \frac{s_{i}}{4 g_{i}\left(a_{k}-\xi_{i}\right)},
$$

являются однозначными и обратными друг $к$ другу, причем имеют место квадратичное и билинейное тохдества

$$
4 \sum_{k} \frac{c_{k}^{2}}{x_{k}^{2}}=\sum_{m} \frac{s_{m}^{2}}{g_{m}}, \quad 4 \sum_{k} \frac{c_{k} c_{k}^{\prime}}{x_{k}^{2}}=\sum_{m} \frac{s_{m} s_{m}^{\prime}}{g_{m}}
$$

әде $\left\{c_{k}^{\prime}\right\} u\left\{s_{m}^{\prime}\right\}-$-ва других вектора, связанных преобразованием (12).

Преобразования (12) напоминают прямое и обратное дискретные преобразования Фурье; ниже воспользуемся ими для анализа рассматриваемых гамильтоновых систем.

В эллиптических координатах кинетическая энергия, импульсы и первые интегралы (1) геодезического потока на эллипсоиде выглядят следующим образом:

$$
T=\sum_{i} \frac{g_{i} \xi_{i}^{2}}{2}=\sum_{i} \frac{\eta_{i}^{2}}{2 g_{i}}, \quad \eta_{k}=g_{k} \dot{\xi}_{k}, \quad f_{k}=x_{k}^{2} \sum_{i} \frac{\eta_{i}^{2}}{g_{i}\left(a_{k}-\xi_{i}\right)} .
$$

Разлагая правые части формул (10) по убывающим степеням $\lambda$ и сравнивая с (9), получаем

ПРЕДЛОЖЕНИЕ 1. Базисные многочлены имеют следующее представление в эллиптических координатах:

$$
u_{k}^{(\nu)}=x_{k}^{2} \sum_{i} \frac{\xi_{i}^{\nu-1}}{4 g_{i}\left(a_{k}-\xi_{i}\right)}, \quad u^{(\nu)}=\sum_{i} \frac{\xi_{i}^{\nu-1}}{4 g_{i}} .
$$

Для гамильтониана и первых интегралов потока $g_{h}^{t}$ в этом случае справедливы равенства

$$
h=\sum_{i} \frac{\eta_{i}^{2}+\xi_{i}^{\nu-1} / 2}{g_{i}}, \quad F_{k}=x_{k}^{2} \sum_{i} \frac{\eta_{i}^{2}+\xi_{i}^{\nu-1} / 2}{g_{i}\left(a_{k}-\xi_{i}\right)} .
$$


Представления типа (13) являются характеристическими для представлений в эллиптических координатах $\left\{\xi_{k}\right\}$ любых решений системы (5). В этих координатах система (5) имеет вид

$$
\frac{\partial\left(u_{k}-x_{k}^{2} u /\left(a_{k}-\xi_{m}\right)\right)}{\partial \xi_{m}}=0 .
$$

С помощью теоремы 5 доказьвается

Теорема 6. Общее решение системы (14) дается формулами

$$
u_{k}=x_{k}^{2} \sum_{i} \frac{v_{i}\left(\xi_{i}\right)}{g_{i}\left(a_{k}-\xi_{i}\right)}, \quad u=\sum_{i} \frac{v_{i}\left(\xi_{i}\right)}{g_{i}}
$$

где $v_{i}$ - произвольные дифференцируемые функиии.

СледствиЕ 1. Система уравнений (5) имеет решениями многочлены тогда и только тогда, когда в представлениях (15) все функиии $v_{i}$ совпадают, $v_{i}\left(\xi_{i}\right)=v\left(\xi_{i}\right)$, иявлятся многочленами.

Возможно, тем же свойством обладают любые рациональные решения системы (5), с той лиш разницей, что $v\left(\xi_{i}\right)$ есть рациональная функция.

СледСтвИЕ 2. Пусть потенциал $u(x)$ в гамильтониане (3) и потенииалы $u_{k}$ в первых интегралах (2) в әллиптических координатах имеют представление (15). Тогда

$$
h=\sum_{i} \frac{\eta_{i}^{2}+2 v_{i}\left(\xi_{i}\right)}{g_{i}}, \quad F_{k}=x_{k}^{2} \sum_{i} \frac{\eta_{i}^{2}+2 v_{i}\left(\xi_{i}\right)}{g_{i}\left(a_{k}-\xi_{i}\right)} .
$$

ПРЕДЛОЖЕНИЕ 2. Преобразованию (7) соответствует преобразование $v_{i}\left(\xi_{i}\right) \rightarrow \xi_{i} v_{i}\left(\xi_{i}\right)$ в представлении (15).

Определим производящую функцию первых интегралов $F_{k}$, имеющих представление (15), равенством

$$
\Phi_{\lambda}(x, p)=\sum_{k} \frac{F_{k}(x, p)}{a_{k}-\lambda} .
$$

Здесь мы следуем Мозеру [2], который точно так же определил производящую функцию интегралов (1). Используя значение производящей функции Мозера, получаем

ПРЕДЛОЖЕНИЕ 3. Для производящей функиии $\Phi_{\lambda}(x, p)$ справедливо равенство

$$
\begin{aligned}
& \Phi_{\lambda}(x, p)=Q_{\lambda}^{2}(x, p)+\left(1-Q_{\lambda}(x)\right)\left(Q_{\lambda}(p)+2 V_{\lambda}(x)\right), \\
& Q_{\lambda}(x, p) \equiv \sum_{i} \frac{x_{i} p_{i}}{a_{i}-\lambda}, \quad V_{\lambda}(x) \equiv \sum_{i} \frac{v_{i}\left(\xi_{i}\right)}{g_{i}\left(\xi_{i}-\lambda\right)} .
\end{aligned}
$$

ТЕОРема 7. Функиии $\Phi_{\lambda}(x, p)$ u $\Phi_{\mu}(x, p)$ коммутируют:

$$
\left\{\Phi_{\lambda}(x, p), \Phi_{\mu}(x, p)\right\}=0 .
$$

Эта теорема обобщает соответствующую теорему Мозера [2] для его производящей функции и доказывается аналогично. 
4. Вдоль траекторий натуральной гамильтоновой системы с потенциалом $u(x)$, имеющего в эллиптических координатах представление (15),

$$
x_{k}^{2} \sum_{i} \frac{\eta_{i}^{2}+2 v_{i}\left(\xi_{i}\right)}{g_{i}\left(a_{k}-\xi_{i}\right)}=F_{k}=\mathrm{const}
$$

(см. формулу (16)). Теорема 5 позволяет разрешить эти соотношения относительно импульсов:

$$
\eta_{k}^{2}+2 v_{k}\left(\xi_{k}\right)=r\left(\xi_{k}\right) \equiv \sum_{i} \frac{F_{i}}{4\left(a_{i}-\xi_{k}\right)} .
$$

Отсюда получаем дифференциальные уравнения траекторий в виде

$$
\dot{\xi}_{k}=\frac{S_{k}\left(\xi_{k}\right)}{g_{k}}, \quad S_{k}\left(\xi_{k}\right) \equiv \sqrt{r\left(\xi_{k}\right)-2 v_{k}\left(\xi_{k}\right)}
$$

Наконец, тождество

$$
\sum_{i} \frac{\xi_{i}^{k-1}}{g_{i} D\left(\xi_{i}\right)}=-4 \delta_{k n}
$$

дает возможность преобразовать уравнения (17) к квадратурам:

$$
\sum_{i} \frac{\xi_{i}^{k-i} \dot{\xi}_{i}}{D\left(\xi_{i}\right) S_{i}\left(\xi_{i}\right)}=-4 \delta_{k n} \Longrightarrow \sum_{i} \int \frac{\xi_{i}^{k-i} d \xi_{i}}{D\left(\xi_{i}\right) S_{i}\left(\xi_{i}\right)}=-4 \delta_{k n}\left(t-t_{0}\right) .
$$

Формулы (18) можно получить также методом Гамильтона-Якоби. В случае полиномиального потенциала $u(x)$ знаменатели подынтегральных выражений в (18) есть радикалы от одинаковых многочленов, т.е. возникает задача Якоби обращения гиперэллиптических интегралов.

Автор благодарит С. Б. Лебле за обсуждение результатов и Ю. Н. Фёдорова за полезные замечания.

Калининградский государственный университет

Поступило

03.12 .92

\section{СПИСОК ЦИТИРОВАННОЙ ЛИТЕРАТУРЫ}

1. Marshall I., Wojcichowsky S. // J. Math. Phys. 1988. V. 29. №6. P. 1338-1346. 2. Мозер Ю. // УМH. 1981. T. 36. № 5. C. 109-151. 3. Garnier R. // Rend. Sirc. Matem. Palermo. 1919. V. 43. №4. P. 155-191. 4. Chudnovsky D. V., Chudnovsky G. V. // C. R. Acad. Sci. Paris. Ser. A. 1978. V. 286. №5. P. 1075-1078. 5. Zaitsev A. A. // Proceedings of the 8th International Workshop Nonlinear Equations \& Dynamical Systems. Need's-92 / ed. V. Makhankov. Singapore: World Scientific, 1993. P. 463-466. 